\title{
Integrative Medizin aus «philosophischer Sicht»
}

Der in der integrativen Medizin eine tragende Rolle spielende Begriff des «Ganzheitlichen» könne leicht missbraucht werden, sei unwissenschaftlich und antiliberal, so Michael Rüegg in einer früheren SÄZ-Ausgabe. Diese Vorwürfe werden hier u. a. mit dem Hinweis zurückgewiesen, dass die Existenz von Ganzheiten ausser Zweifel stehe und diese von rein kausal-mechanistischen Schlussfolgerungen nicht erfasst werden könnten.

Matthias Wiesmann

\footnotetext{
* Rüegg M. Grenzen aus philosophischer Sicht. Schweiz Ärztezeitung. 2012;93(22):826-9.
}

Korrespondenz: Matthias Wiesmann M.A. Oberkirchstrasse 15 CH-8500 Frauenfeld

\section{Die drei Voten des Michael Rüegg}

Am 18. und 19. Januar 2012 veranstaltete das Departement Pflege des Kantonsspitals St. Gallen die Tagung «Integrative Medizin und Pflege - mehr als ein Trend». Hier hielt lic. phil. Michael Rüegg ein Referat zum Thema «Integrative Medizin und Pflege an öffentlichen Spitälern - Grenzen aus philosophischer Sicht». Dieses Tagungsreferat hatte ein Vorund ein Nachspiel: Unter der etwas weniger philosophischen Headline «Ganzheitlichkeit - ein Abgrund?» veröffentlichte das St. Galler Tagblatt am 17. Januar 2012 ein Interview mit Michael Rüegg. Während Rüegg auf der Website der Veranstalter (www.fachsymposium.ch) im Unterschied zu den meisten anderen Referentinnen und Referenten keine Referatzusammenfassung hinterlassen hatte, veröffentlichte die Schweizerische Ärztezeitung eine «überarbeitete Fassung» [1]. In der Autorennotiz wird Rüegg mit den Attributen «Doktorand im Bereich Philosophie» und «Experte für Komplementärmedizin» versehen. Dies und das Referatthema wecken Erwartungen, namentlich die Erwartung, dass da jemand philosophisch-konsequent Prämissen und Folgerungen befragt und stringent argumentiert. Bei genauerer Betrachtung entpuppt sich die «überarbeitete Fassung» streckenweise als Kompilat des Zeitungsinterviews. Sehen wir uns die tiefoder abgründige Argumentation näher an.

\section{Worum geht es dem Referenten?}

Rüeggs Anliegen lässt sich folgendermassen zusammenfassen:

Integrative Medizin arbeitet mit dem Begriff der Ganzheitlichkeit. Ganzheitlichkeit sei nicht nur kein wissenschaftlich brauchbarer Begriff und infolgedessen Weltanschauung, sondern er sei zudem tendenziell totalitär und antiliberal. Ärzte, die auf dieser Grundlage arbeiten, gäben sich ihren Patienten gegenüber als wissenschaftlich und machten sich damit der Täuschung schuldig. Rüegg nimmt an weiteren Aspekten Anstoss. Ich möchte mich - wie ja auch Rüegg im Tagblatt - auf das Thema Ganzheitlichkeit beschränken.

\section{Wo sieht Rüegg Probleme mit «Ganzheitlichkeit»?}

Unproblematisch sei sie da, wo weniger spezialisierte Herangehensweisen von spezialisierten unterschieden werden - z. B. diejenige von Hausärzten gegenüber derjenigen von Spezialärzten. Problematisch sei der Begriff hingegen dort, wo ein wissenschaftlicher Anspruch (bzw. Anmassung) vorliege, man verfüge über die ganzheitliche Sicht. Einverstanden. Ein gesundes Misstrauen ist immer angebracht, wenn jemand beansprucht, die einzig gültige Erkenntnismethodik zu vertreten - Rüegg und Hochschulmedizin eingeschlossen. Wie argumentiert Rüegg? Per Analogieschluss kommt er zum Marxismus-Leninismus. Dieser beanspruche ja auch eine wissenschaftliche Gesamtsicht. «Der Duden», der in Rüeggs philosophischem Handapparat offenbar griffbereit steht, sagt ihm nun, dass «totalitär» «selten für ganzheitlich» stehe. Nun stellt Rüegg Ganzheitlichkeit definitiv (nicht nur selten) in die totalitäre, antiliberale Ecke. Dass man Ärzte mit einer solchen Geisteshaltung nicht auf Patienten in Spitälern loslassen darf, ist naheliegend. Weniger klar ist, welche philosophische Methodik dieser Abfolge von Rüeggs Sätzen zugrunde liegt.

\section{Empiristische Naturwissenschaft ...}

Hätte Rüegg recht mit seinem Brückenschlag von Medizinern mit Ganzheitlichkeitsambitionen zu Totalitarismus, müsste man ihm zustimmen. Bevor derart gravierende Urteile ausgesprochen werden, müsste aber doch eine einigermassen sorgfältige Bestandsaufnahme erfolgen. Es darf oder soll durchaus eine philosophisch-erkenntniskritische sein.

Die heute dominante wissenschaftliche Methodik (Empirismus oder wie auch immer genannt) führt zwangsläufig dazu, dass einzelne Variablen aus einem Lebenszusammenhang isoliert und unter- 
sucht werden. Jede andere Vorgehensweise ist ideologieverdächtig. Übersprungen wird dabei in der Regel die Problematik, dass bereits die einfachste Aussage, die eine Wahrnehmung beinhaltet, ein Urteil ist. Die Wiener Schule des Positivismus versuchte, diese Problematik mit «Protokollsätzen» zu bewältigen. Im Wissenschaftsalltag wird Messbarkeit vorausgesetzt, was in Bezug auf die Unendlichkeit an möglichen Protokollsätzen einer massiven Einschränkung gleichkommt. (Einem Phänomen wie «Schmerz» kommt keine messbare Realität zu.) Somit unterbleibt ein umfassender (ganzheitlicher) Blick auf Zusammenhänge. Die Forderung nach Messbarkeit und das Denken in Kausalitäten verhindern, dass die Vielfalt der Wirklichkeit erfasst werden kann.

Auch wenn Zusammenhänge zu kurz kommen, ist es keine Frage, dass die an Messbarem orientierten Wissenschaften ein enormes Wissen über Natur und Mensch zusammengetragen haben, das erlaubt, wirkungsvoll in Zusammenhänge einzugreifen. Es ist selbstverständlich nützlich (um dies mit einer Analogie anschaulich zu machen), bei einem Leitungsbruch genaue Kenntnis des Leitungsnetzes zu haben, um mit einer Unterbrechung am richtigen Ort grösseren Schaden verhindern zu können. Die Orientierung an kausalen Zusammenhängen und quantitativer Erfassung kommt der technologischen Verwertung entgegen.

\section{... mit Nebenwirkungen}

Allerdings zeigt die Geschichte, dass einfache Eingriffe in komplexe Wirkungszusammenhänge zwar immer wieder beabsichtigte Wirkungen zeigten,

\section{Der Weltanschauungscharakter der empiristischen Naturwissenschaft}

Trotz offensichtlichen Unzulänglichkeiten hat sich in den Hochschulwissenschaften die Überzeugung (der Glaube) behauptet, dass die Welt auch allein mit kausal-mechanistischen Erklärungen ausreichend zu verstehen sei. Wo komplexere Zusammenhänge so nicht verstanden werden können (was ist Leben?), wird kein prinzipielles Unvermögen eingeräumt, sondern man schiebt das Erkenntnisproblem hinaus und sagt «noch nicht». Man glaubt, auf diesem Weg eines Tages zu umfassenden Erklärungen zu kommen. Tatsächlich handelt es sich beim kausal-mechanistischen Erklärungsversuch also um Glauben, um eine Weltanschauung. Es handelt sich um ein Bekenntnis zu einem Funktionsmodell der Welt. Wer diesen Bekenntnischarakter ausblendet und beansprucht, über den alleingültigen Erkenntnisweg zu verfügen, landet unweigerlich in der totalitären Ecke. Misstrauen ist angebracht (siehe oben). Der Balken im eigenen Auge wird von den Vertretern dieses Ansatzes geflissentlich übersehen: Für sie gibt es weltanschaulich beeinflusste Wissenschaft und ihre eigene «richtige» Wissenschaft, die sich aber eben immer mehr oder weniger explizit zu einer reduktionistischen, kausal-mechanistischen Weltanschauung bekennt, die kausalen Zusammenhänge aber nie (ausser allenfalls im Bereich der unbelebten Natur) nachweisen kann. (Auch dort sind die Beweise in der Regel statistischer Natur; Korrelationen sind keine Kausalitäten.)

Eine ordentliche Portion Weltanschauung steckt in den Naturwissenschaften nicht nur aufgrund der primär auf kausal-mechanische Zusammenhänge

\section{«Übersprungen wird dabei in der Regel die Problematik, dass bereits die einfachste Aussage, die eine Wahrnehmung beinhaltet, ein Urteil ist.»}

gleichzeitig aber nicht beabsichtigte und nicht vorausgesehene Nebenwirkungen zur Folge hatten und haben. So ist es nicht erstaunlich, dass beispielsweise eines Tages damit begonnen wurde, ein Insektizid namens DDT einzusetzen - mit den bekannten dramatischen Folgen des «Stummen Frühlings». Noch heute werden in der Tierfütterung oder beim Feuerbrand recht unbekümmert flächendeckend Antibiotika eingesetzt, was zu fatalen Resistenzen führt. Ins oben skizzierte Beispiel des Leitungsnetzes übertragen: Symptome wie die Wasserfontäne wurden zum Verschwinden gebracht, der Bruch wird allenfalls behoben, jedoch nicht die Randbedingungen, die dazu geführt haben. fokussierenden Forschungsfragen. Analoge Modelle oder Bilder dominieren auch das - in unserem Fall physiologische oder medizinische Alltagsdenken. «Das Herz ist eine Pumpe» weist primär auf ein Modell oder Bild einer Funktion unseres Organismus hin. Es handelt sich nicht um eine umfassende Beschreibung eines Organs in seinen Wechselwirkungen mit den vielgliedrigen Funktionen unseres Gesamtorganismus. Der weltanschauliche Charakter dieses Bildes steckt in der Maschinenanalogie. Unbekümmert um den weltanschaulichen Charakter des eigenen Erkenntnisansatzes verlangt die Hochschulmedizin (bzw. das Kartell von Hochschulmedizin und Gesundheitsbehörden), dass sich die Komplementärmedizin dem «Goldstandard» der Hochschulmedizin unterziehe. Erkenntnisse aufgrund 


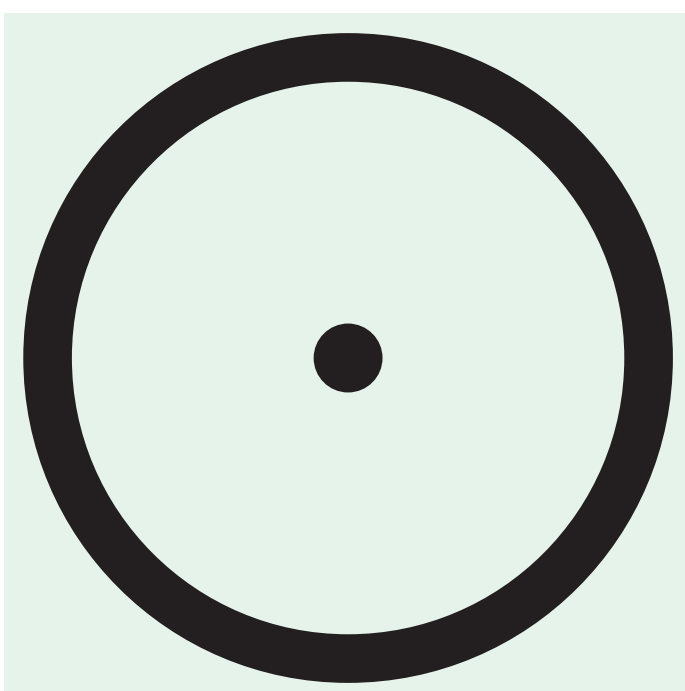

Ganzheit: Nur mit kausal-mechanistischen Methoden der Naturwissenschaft kann sie nicht erfasst werden; dies zu behaupten, ist Weltanschauung.

qualitativer Verfahren werden a priori als unwissenschaftlich zurückgewiesen.

\section{Gegenbewegung}

Der «stumme Frühling» führte partiell allerdings zu einem Gesinnungswandel, der in Ansätze der Systemtheorie, Kybernetik, der Ökologie, Psychosomatik oder anderen Spielarten von Herangehensweisen, die Ganzheitlichkeit anstreben, münden. Der Philosoph Edmund Husserl versuchte mit der «Epoché» eine Methode zu entwickeln, die ebenfalls in einem ganzheitlichen Erkenntnisvorgang das Wesen des Erkenntnisgegenstandes erschliessen sollte. Der Wunsch nach ganzheitlichem Erfassen von Zusammenhängen kann wissenschaftssoziologisch auch als eine Reaktion auf den kausal-mechanistischen Approach in den meisten Wissenschaften angesehen werden.

Solche knüpfen an im 19. Jahrhundert entwickelte Ansätze an, die von den Erfolgen der nach kausalen Abhängigkeiten von zwei oder mehr Variablen Ausschau haltenden, technologisch problemlos verwertbaren Wissenschaft verdrängt wurden. Ein Anknüpfungspunkt ist der morphologische Ansatz u. a. von Goethe. Dass es so etwas wie Ganzheiten tatsächlich gibt, steht ausser Zweifel. Ganzheit hat beispielsweise im Auge, wer von System spricht. Ein Problem für ihn ist allenfalls die Frage der Systemgrenzen. Ohne eine ordentliche Portion morphologischer Sichtweise wäre es beispielsweise nicht möglich, überhaupt von Pflanze, Tier oder Mensch zu sprechen. Irgendwie gehören ja die wahrgenommenen Einzelerscheinungen zu einem Ganzen. Das dürfte auch von einem eingefleischten Empiristen auf einer - wie er es vielleicht bezeichnen würde vorwissenschaftlichen Ebene nicht bezweifelt werden. Erkenntnisentwicklungen in der ersten Hälfte des letzten Jahrhunderts, die mit Typenbildungen arbeiteten (Soziologie: Max Weber; Biologie: Adolf Portmann) gingen ebenfalls von Ganzheiten aus. Ob von Typus oder Gestalt die Rede ist: Immer handelt es sich um Bildbegriffe. (Ein Bild verwendet auch, wer das Herz als Pumpe bezeichnet!)

\section{Erkennen von und mit Bildern}

Bilder sind wichtig in einer Betrachtungsweise von lebenden Organismen, die sich um Ganzheitlichkeit bemüht. Nur wird hier versucht, sachgemässe Bilder zu entwickeln und nicht einfach mechanistische Vor-Bilder zu verwenden. Nicht Kausalitäten oder Funktionalitäten («weil» / «um zu») stehen im Vordergrund, sondern die Beschreibung der Gestalt.

Gelegentlich lassen sich Ergebnisse bildbeschreibender Erkenntnismethoden auch empirisch-statistisch nachvollziehen. Dazu das Beispiel einer sogenannt bildschaffenden (nicht bildgebenden!) Methode: Wenn organische Substanzen in eine Kupferchloridlösung gegeben werden und diese auskristallisiert, entstehen typische Kristallisationsbilder. In verblindeten Versuchen wurde nachgewiesen, dass mit dieser Methode beispielsweise Getreidekörner aus biologischer und aus konventioneller Produktion unterschieden werden können. Hier ist es die Gestalt, die «gelesen» wird (ein wissenschaftlich erlernbares Werkzeug, wie Rüegg es fordert), es handelt sich nicht um eine statistische Abhängigkeit, die berechnet wird. (Der empirisch-statistische Nachvollzug ist allerdings nicht als Wahrheitsbeweis zu verstehen.)

Die Gestaltpsychologie zeigt, dass der Erkenntnisvorgang im Alltag keineswegs entlang den Strukturen kausal-mechanistischer Schlussfolgerung verläuft. Sicherlich auch im diagnostischen Vorgang eines Arztes nicht. So würde er wohl nur dann vorgehen, wenn er wie eine Software funktionieren würde, die seine von ihm eingegebenen Hardfacts zu einer Diagnose verarbeitet, während er selber sich jeden Urteils enthalten würde. Der Mensch ist in der Lage, Wahrnehmungen sehr schnell in Ordnungen, Bildern oder eben einer Gestalt zu strukturieren. In diesem Sinn ist es sehr weise, dass man auch in der Medizin von Krankheitsbild spricht. Jedes Bild konstituiert sich aus Einzelwahrnehmungen, aus einzelnen Bildelementen.

Beim Erlernen des Erkennens von Gestalt, die nicht nur in raum-zeitlichen Kategorien zu erfassen ist, sondern auch viele qualitative Elemente umfasst, handelt es sich ebenso um ein Studium wie beim Erlernen beispielsweise apparativer Diagnostik. Einer ganzheitlich orientierten Wissenschaft geht es darum, Gestalt bereits in der wissenschaftlichen Beschreibung der Wirklichkeit zu finden.

Damit sind einige erkenntnismethodische Aspekte angedeutet, die in einer sorgfältigen philosophischen bzw. erkenntniskritischen Betrachtung zu beachten wären. Eine Abhandlung, die den reduktionistischen Ansatz implizit absolut setzt, hat eher totalitären als philosophischen Charakter. 